\title{
BIRDS OF JOYPURHAT DISTRICT, BANGLADESH
}

\author{
Shapna Karmakar, Selina Parween and A. M. Saleh Reza
}

\author{
Department of Zoology, University of Rajshahi, Rajshahi 6205, Bangladesh \\ *Corresponding author (email: parween_s@yahoo.com)
}

\begin{abstract}
With a view to gather information on the diversity, habitat, distribution and residential status of avifauna of Joypurhat District under Rajshahi Division, a systematic field study was conducted for a period of eight months from April to November 2010. The study area was divided into the following five Upazila sites viz. Site A Joypurhat Sadar; Site B Khetlal; Site C Panchbibi; Site D Akkelpur and Site E Kalai. A total of 57 species, belonging to 8 orders, 28 families and 48 genera, were recorded. Of 57 species, $47.37 \%$ were non-passerines and $52.63 \%$ passerines; $89.43 \%$ were resident and $11.57 \%$ migratory. The status of the birds ranged from $14.04 \%$ for fairly common to $45.61 \%$ for rare species. The diversity of the bird species ranged from $25(47.37 \%)$ for site D to $39(68.42 \%)$ for site A. The study revealed that most of the species used more than one habitat and the highest number of species, 52 (91.23\%), was observed on the trees, whereas the lowest number of species, 3 (5.26\%), was found around the ponds. A number of environmental and man-made factors appeared to be responsible for declining population and species diversity of the birds in the study area.
\end{abstract}

Key-words: Bird diversity, Joypurhat District, resident, migratory, passerine.

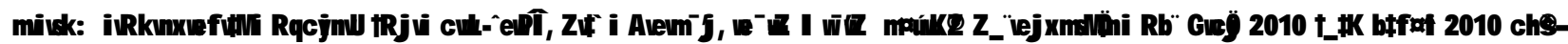

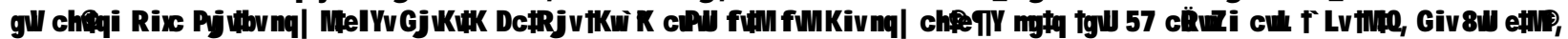

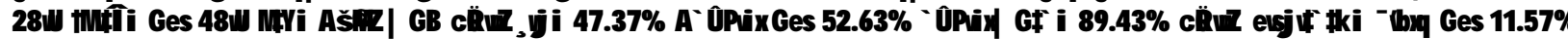

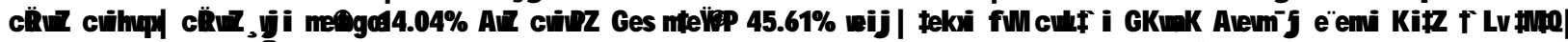

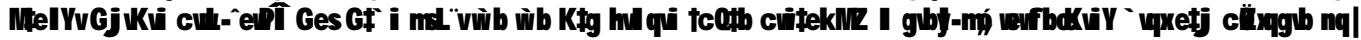

\section{Introduction}

The national list of the birds of Bangladesh owns as many as 650 species (Siddiqui et al. 2008), of which, 620 species have been recorded in recent years and can be asserted to be present today. Out of these 143 are vagrant (occurring very irregularly or seen extremely rare) in the country, and the rest 477 are regular species. Among the regular species, 301 are residents and 176 are seasonal visitors to Bangladesh. Out of 176 regularly occurring migratory species, 160 are winter visitors, six are summer visitors and 10 are spring or passage migrants. According to the relative abundance, Bangladesh has 143 vagrants, 176 rare, 103 uncommon and 198 common species of birds. Among the total observed birds, 40 species are globally threatened or nearly threatened, and a further 13 species are globally threatened and by now have been extirpated in Bangladesh (Banglapedia 2003). However, recent information reports 736 species of birds in the country (Khan 2008a).

For community study of flora and fauna, species diversity is a function of the number of different species, the number of individuals per species, and the total number of individuals of all species present in that community at a definite time period (Southwick 1976).
It is a challenging parameter to measure because diversity is organized hierarchically, and as a consequence, pattern of diversity at one level is linked statistically and by evolutionary history of the patterns of higher and lower levels (Gottelli 2002). Out of the total number of species in the trophic component, or in a community as a whole, relatively small percentages are usually abundant and a large percentage is rare. While the few common species, or dominants, largely account for the energy flow in trophic group, it is the large number of rare species that largely determine the species diversity of trophic groups and whole communities (Odum 1971). Abundance and distribution is one of the major characteristics of species in a community. It indicates the status of particular species and shows the ecological conditions of a particular environment.

The bird populations of Bangladesh are decreasing at an alarming rate like elsewhere in the world mainly due to habitat destruction with the subsequent effect on food and shelter (Hussain 2008). Although birds play a crucial economic role in pest control, scavenging and pollinating as well as providing food to mankind, they are highly susceptible to any changes in the habitat they live, and their diversity is decreasing at a considerable rate because of human unconsciousness for their 
protection and conservation (Sarker et al. 2001). Reports on avifauna in different regions of Bangladesh have been published. These include, among others, Hussain (1969, 1975) in Chittagong Hill Tracts and Pablakhali Wildlife Sanctuary, Haque (1976) at the Rajshahi University Campus, Khan (1982) and Sarker et al. (2001) in St. Martin Island, Sarker and Sarker (1986) in the Sundarbans, Sarker et al. (2000a, b) at Bagkhali range, Cox's Bazar and Moheskhali island, respectively, and Sarker et al. (2009) at two sites of the Uttara Model Town, Dhaka. A literature survey shows that no recent work has been done on the avifauna of Joypurhat and its adjacent areas. The present work therefore was undertaken to describe the existing bird fauna of Joypurhat District with special reference to their habitat, abundance and residential status, which will provide some basic information for future in-depth studies and conservation of the birds in the study area.

\section{Methodology}

Study area: Joypurhat is a District of Rajshahi Division that has an area of 965.44 sq. $\mathrm{km}$. It is bounded by Dinajpur in the north, Naogaon in the south, and Bogra and Gaibandha in the east, Bogra and Naogaon and West Bengal, India in the west (Banglapedia 2003). For the convenience of the field work, the study area was divided into five sites on the basis of administrative Upazilas as follows: Site A Joypurhat Sadar, Site B Khetlal, Site C Panchbibi, Site D Akkelpur and Site E Kalai ( Fig. 1).

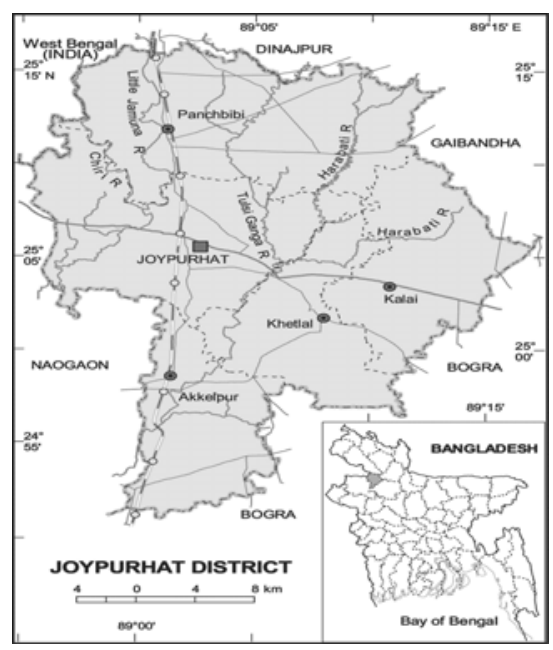

Fig. 1. Map showing five sites $(\bullet)$ of the study area.

Observation schedules: Observations were made fortnightly in the mornings during summer, winter and monsoon (when there was no rain). Occasionally field visits were carried out in the afternoons. At least two monthly visits were carried out at each site of the study area. However, from mid April to end of May 2010, three visits were made per week. A special visit was made to Site B (i.e. Khetlal Upazila) where a heronry is situated and protected by the local people. The study was conducted for a period of eight months from April to November 2010 in Joypurhat District under Rajshahi Division, located at the northern region of Bangladesh.

Observation methodology: Mainly eye observations were made to watch the birds. Often a binocular was used to locate the bird at a distance and to observe them more clearly. A digital camera (Olympus FE-20) was used to take photographs of the birds.

Vegetation of the study area: Bangladesh has been tentatively divided into 30 agroecological zones. The study area comes under Level Barind Tract where the landscape is almost plain. The predominant soils have grey, silty, puddled topsoil with ploughpan. The soils are low in available moisture holding capacity and slightly acidic to acidic in reaction. Organic matter status is very low and most of the available nutrients are limited. So, the vegetation of the study area is well planted, comprising both indigenous and exotic species. The predominant vegetation of the study area is composed mainly of woody trees. The major tree species are Kanthal (Artocarpus hetarophyllus), Aam (Magnifera indica), Jam (Syzygium cumini), Pitraj (Aphanamixis polystachya), Mehogoni (Swietenia mehagoni), Bot (Ficus benghalensis), Eucalyptus (Eucalyptus spp.), Akashmoni (Acacia moniliformis), Boroi (Zizyphus mauritiana), Koroi (Albizzia spp.), Shimul (Salmalia spp.) and Coconut (Cocos nucifera) The study area is composed of both urban and rural populations but the rural area contains much plant diversity than that of the urban area.

Species identification: The birds were mainly identified with the help of their photographs using two famous books by Ali (1996) and Hussain (2008). The common English, scientific and vernacular names of the birds have been taken from Ali (1996), Grewal et al. (2002), Harvey (1990) and Khan (2008a, b). The following three characteristics were applied to identify the bird species.

External morphology: Colour, shape, size, beak, leg and tail of the birds were the most important features to identify the species. 
Song and calls: Identification was also made on the basis of songs or/and calls of the birds. This was mainly assisted by the people residing in the study area.

Habitats: The species were also identified by observing the habitats of the birds that fall under eight categories as follows: 1. open area, 2. agricultural land, 3. human habitations, 4. bush, 5. tree, 6. pond, 7. ditch and 8. other water bodies.

Status justification: The following status of birds was justified on the basis of the standard methods used by Khan (1982).

(i) Very common (VC): Species those were found almost allover the study area and were recorded in 80$100 \%$ field visits/working days.

(ii) Common (C): Species those were recorded in 5079\% field visits/working days.

(iii) Fairly common (FC): Species those were recorded in $20-49 \%$ in two or three sites of the study area during the field visits; and

(iv) Few or rare (R): Specie those were seen rarely in one or two sites of the study area in less than 19\% during the visits.

The status of the birds was calculated in terms of percentage of occurrence.

\section{Results and Discussion}

A total of 57 species of birds was recorded from the study area (Table 1). They were found to belong to nine orders, 26 families and 48 genera, comprising 23 passerines and 25 non-passerines. The resident birds constituted $50(87.72 \%)$ species while the migratory ones had 7 (12.28\%), suggesting that the resident species were nearly 7 times higher than those of the migratory species. The migratory species are Clamator jacobinus (Chokhgelo), Eudynamys scolopaceus (Kalo Kokil), Actitis hypoleucos (Cha-pakhi), Amaurornis phoenicurus (Dahuk), Milvus migrans (Bhubon Chil), Motacilla alba (Sada Khonjan), and Motacilla flava (Halud Khanjan). Two resident and FC birds are shown in Plate 1.

The highest number of 30 species (52.63\%) and 22 genera (45.83\%) belonged to the order Passeriformes and a single species (1.75\%) and single genus (2.08\%) belonged to the order Gruiformes. The family Corvidae (Order Passeriformes) included the highest 10 species (i.e., $17.54 \%$ of the total and $33.33 \%$ of the passerines) and the highest 7 genera (i.e., $14.85 \%$ of the total and $30.43 \%$ of the passerines).
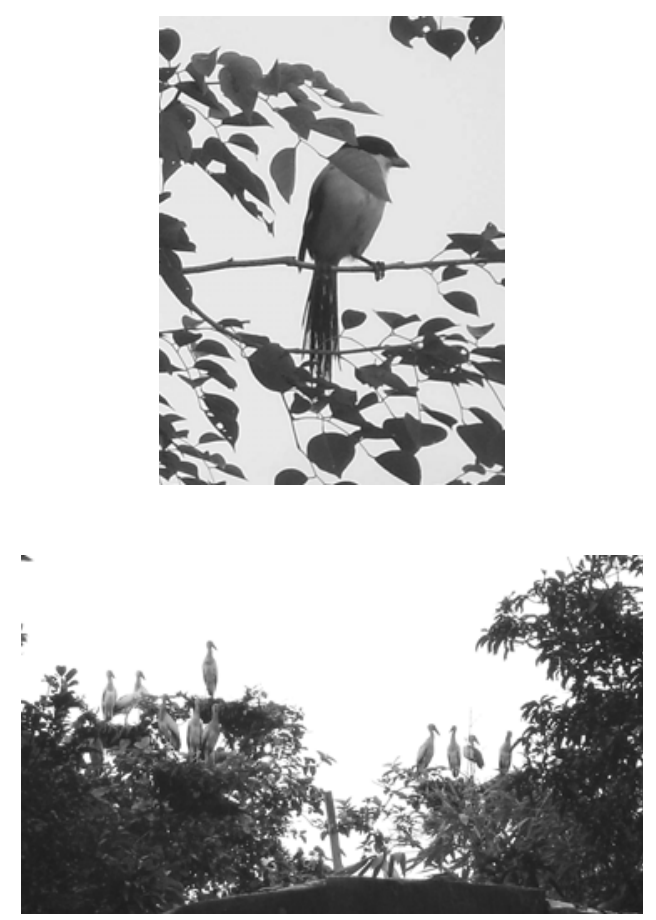

Plate 1. (Above) Rufous Backed Shrike (Lanius schach), (below) Asian Open bill Stork (Anastomus oscitans

A total of 14 families, 9 non-passerines and 5 passerines, had only a single species. Of the 48 genera, a single genus Dicrurus (Passeriformes: Corvidae) contained the highest number of species (3 i.e., 6.25\% of the total and $13.04 \%$ of the passerines).

Out of 25 non-passerine genera, the Family Ardeidae contained 4 genera $(8.33 \%$ of the total and $16 \%$ of the non-passerines). Dinopium (Piciformes: Picidae) and Merops (Coraciformes: Meropidae) had 2 species each that represented $3.51 \%$ of the total and $7.41 \%$ of the non-passerines.

The availability status of the recorded species is shown in Table 1 \& 2. It varied from 8 for FC (14.04\%) to 26 for R (45.61\%). Percentage of $\mathrm{C}$ species (13 in number, $22.81 \%$ ) was higher than that of VC species (10 in number, 17.54\%). Moreover, the number of VC species was the same for both the non-passerine and passerine birds. The status of all 6 migratory species was R.

The results on species diversity among different sites of the study area are presented in Table 3. The diversity varied from 25 (47.37\%) for Site D to 39 (68.42\%) for Site A. The second highest value was recorded from Site B, 37 (64.91\%). The species diversity was found to 
be similar for Sites $\mathrm{C}$ and $\mathrm{D}$. The highest species diversity in Site A might be due to such factors as the increase in species conservation intensity, higher tree plantation activities and re-habilitation of the bird species. The lowest species diversity in Sites C an D, on the other hand, might be due to the felling of forest cover and man's deliberate actions.

Table 1. A systematic list of birds of Joypurhat District, Bangladesh.

\begin{tabular}{|c|c|c|c|c|c|}
\hline $\begin{array}{l}\text { Orders } \\
\text { (Families) }\end{array}$ & Scientific Names & English Names & Bangla Names & $\begin{array}{l}\text { Country } \\
\text { Status* }\end{array}$ & $\begin{array}{l}\text { Status of } \\
\text { Abundance** }\end{array}$ \\
\hline \multirow{3}{*}{$\begin{array}{l}\text { Piciformes } \\
\text { Picidae }\end{array}$} & $\begin{array}{l}\text { Dendrocopos macei } \\
\text { (Vieillot 1818) }\end{array}$ & $\begin{array}{l}\text { Fulvous-breasted Pied } \\
\text { woodpecker }\end{array}$ & Pakra Kaththokra & $\mathrm{R}$ & $\mathrm{R}$ \\
\hline & $\begin{array}{l}\text { Dinopium javanense } \\
\text { (Liungh 1797) }\end{array}$ & $\begin{array}{l}\text { Indian Golden-backed } \\
\text { woodpecker }\end{array}$ & Pati Kaththokra & $\mathrm{R}$ & $\mathrm{VC}$ \\
\hline & $\begin{array}{l}\text { Dinopium benhgalense } \\
\text { (Linnaeus 1758) }\end{array}$ & $\begin{array}{l}\text { Lesser Golden-backed } \\
\text { woodpecker }\end{array}$ & Bangla Kaththokra & $\mathrm{R}$ & $\mathrm{VC}$ \\
\hline $\begin{array}{c}\text { Coraciiformes } \\
\text { Alcedinidae }\end{array}$ & $\begin{array}{l}\text { Alcedo atthis (Linnocus } \\
\text { 1758) }\end{array}$ & Common Kingfisher & $\begin{array}{l}\text { Pati/Choto } \\
\text { Machranga }\end{array}$ & $\mathrm{R}$ & $\mathrm{C}$ \\
\hline \multirow{2}{*}{ Halcyonidae } & $\begin{array}{l}\text { Halcyon smyrnensis } \\
\text { (Linnaeus, 1758) }\end{array}$ & $\begin{array}{l}\text { White-throated / White- } \\
\text { breasted Kingfisher }\end{array}$ & $\begin{array}{l}\text { Sadagola } \\
\text { Machhranga }\end{array}$ & $\mathrm{R}$ & $\mathrm{C}$ \\
\hline & $\begin{array}{l}\text { Todiramphus Chloris } \\
\text { (Boddart, 1783) }\end{array}$ & $\begin{array}{l}\text { Collared kingfisher, } \\
\text { white collared kingfisher }\end{array}$ & $\begin{array}{l}\text { Sada-ghar } \\
\text { Machranga }\end{array}$ & $\mathrm{R}$ & $\mathrm{R}$ \\
\hline \multirow[t]{2}{*}{ Meropidae } & $\begin{array}{l}\text { Merops orientalis } \\
\text { (Latham, 1802) }\end{array}$ & Green Bee-eater & $\begin{array}{l}\text { Choto Suichora, } \\
\text { Banspati }\end{array}$ & $\mathrm{R}$ & $\mathrm{C}$ \\
\hline & $\begin{array}{l}\text { Merops Philippinus } \\
\text { (Linnaeus, 1766) }\end{array}$ & Blue tailed Bee-eater & Nil-lej Suichora & $\mathrm{R}$ & $\mathrm{VC}$ \\
\hline \multirow{3}{*}{$\begin{array}{l}\text { Cuculiformes } \\
\text { Cuculidae }\end{array}$} & $\begin{array}{l}\text { Clamator jacobinus } \\
\text { (Boddaert, 1783) }\end{array}$ & Pied/Pied-crested Cuckoo & $\begin{array}{l}\text { Chokhgelo-Pakhi, } \\
\text { Papya }\end{array}$ & $\mathrm{M}$ & $\mathrm{VC}$ \\
\hline & $\begin{array}{l}\text { Cuculus micropterus } \\
\text { (Gould, 1838) }\end{array}$ & Indian cuckoo & Bau-Kawtha-Kau & $\mathrm{R}$ & $\mathrm{R}$ \\
\hline & $\begin{array}{l}\text { Eudynamys scolopaceus } \\
\text { (Linnaeus,1758) }\end{array}$ & $\begin{array}{l}\text { Asian koel, Asian } \\
\text { Cuckoo }\end{array}$ & Kokil, Kalo Kokil & $\mathrm{R}$ & $\mathrm{VC}$ \\
\hline Centropodidae & $\begin{array}{l}\text { Centropus bengalensis } \\
\text { (Gmelin, 1788) }\end{array}$ & Lesser coucal & Choto Kanakuka & $\mathrm{R}$ & $\mathrm{R}$ \\
\hline $\begin{array}{l}\text { Psittaciformes } \\
\text { Psittacidae }\end{array}$ & $\begin{array}{l}\text { Psittacula krameri } \\
\text { (Scopoli 1769) }\end{array}$ & Rose-ringed Parakeet & Tia, Tota & $\mathrm{R}$ & FC \\
\hline \multirow{2}{*}{$\begin{array}{l}\text { Apodiformes } \\
\text { Apodidae }\end{array}$} & $\begin{array}{l}\text { Cypsiurus balasiensis } \\
\text { (J.E. Gray 1829) }\end{array}$ & $\begin{array}{l}\text { Asian palm swift, palm } \\
\text { swift }\end{array}$ & Nakkati & $\mathrm{R}$ & $\mathrm{C}$ \\
\hline & Apus affinis (J.E.Gray 1830) & House Swift & Ababil & $\mathrm{R}$ & FC \\
\hline \multirow{2}{*}{$\begin{array}{l}\text { Columbiformes } \\
\text { Columbidae }\end{array}$} & $\begin{array}{l}\text { Columba livia } \\
\text { (Gmelin } 1789\end{array}$ & $\begin{array}{l}\text { Rock Pigeon, Blue Rock } \\
\text { Pigeon }\end{array}$ & Jalali Kobutar, Paira & $\mathrm{R}$ & $\mathrm{C}$ \\
\hline & $\begin{array}{l}\text { Streptopelia chinessis } \\
\text { (Scopoli, 1786) }\end{array}$ & Spotted Dove & Tila Ghughu & $\mathrm{R}$ & $\mathrm{C}$ \\
\hline $\begin{array}{l}\text { Gruiformes } \\
\text { Rallidae }\end{array}$ & $\begin{array}{l}\text { Amaurornis phoenicurus } \\
\text { (Pennant, 1769) }\end{array}$ & White-breasted Waterhen & Dahuk & $\mathrm{R}$ & $\mathrm{R}$ \\
\hline $\begin{array}{l}\text { Ciconiiformes } \\
\text { Scolopacidae }\end{array}$ & $\begin{array}{l}\text { Actitis hypoleucos } \\
\text { (Linnaeus, 1758) }\end{array}$ & Common Sandpiper & Chapakhi & $\mathrm{M}$ & $\mathrm{R}$ \\
\hline \multirow[t]{2}{*}{ Accipitridae } & $\begin{array}{l}\text { Milvus migrans } \\
\text { (Boddaert, 1783) }\end{array}$ & Black Kite & Bhubon Chil & $\mathrm{M}$ & $\mathrm{R}$ \\
\hline & $\begin{array}{l}\text { Spilornis cheela } \\
\text { (Latham, 1790) }\end{array}$ & Crested Serpent Eagle & $\begin{array}{l}\text { Tila Baj, Shapkheko } \\
\text { Baj }\end{array}$ & $\mathrm{R}$ & $\mathrm{R}$ \\
\hline \multirow{3}{*}{ Ardeidae } & $\begin{array}{l}\text { Ardeola grayii (Scopoli, } \\
\text { 1832) }\end{array}$ & Pond Heron & Kani/Kana bok & $\mathrm{R}$ & $\mathrm{C}$ \\
\hline & $\begin{array}{l}\text { Egretta garzetta } \\
\text { (Linnaeus, 1766) }\end{array}$ & Little Egret & Choto Bok & $\mathrm{R}$ & $\mathrm{R}$ \\
\hline & $\begin{array}{l}\text { Nycticorax nycticorax } \\
\text { (Linnaeus, 1758) }\end{array}$ & Night Heron & Nishi Bok & $\mathrm{R}$ & FC \\
\hline Ardeidae & $\begin{array}{l}\text { Ixobrychus cinnomomeus } \\
\text { (Gmelin 1789) }\end{array}$ & $\begin{array}{l}\text { Cinnamon Bittern, } \\
\text { Chestnut Bittern }\end{array}$ & Lal Bok, Khairi Bok & $\mathrm{R}$ & $\mathrm{R}$ \\
\hline Ciconiidae & $\begin{array}{l}\text { Anastomus oscitans } \\
\text { (Boddaert, 1873) }\end{array}$ & Asian Openbill Stork & $\begin{array}{l}\text { Shamukh Bhanga, } \\
\text { Shamukh Khol }\end{array}$ & $\mathrm{R}$ & $\mathrm{R}$ \\
\hline
\end{tabular}




\begin{tabular}{|c|c|c|c|c|c|}
\hline $\begin{array}{c}\text { Orders } \\
\text { (Families) }\end{array}$ & Scientific Names & English Names & Bangla Names & $\begin{array}{l}\text { Country } \\
\text { Status* }\end{array}$ & $\begin{array}{l}\text { Status of } \\
\text { Abundance** }\end{array}$ \\
\hline Threskiornithidae & $\begin{array}{l}\text { Pseudibis Papillosa } \\
\text { (Temminck,1824) }\end{array}$ & Black Ibis & $\begin{array}{l}\text { Kalo Duchora / } \\
\text { Kastechora }\end{array}$ & $\mathrm{R}$ & $\mathrm{R}$ \\
\hline $\begin{array}{l}\text { Passeriformes } \\
\text { Irenidae }\end{array}$ & $\begin{array}{l}\text { Chloropsis } \\
\text { cochinchinensis } \\
\text { (Gamelin 1789) }\end{array}$ & Golden fronted leaf bird & $\begin{array}{l}\text { Shonakopali } \\
\text { Horbola, Choto } \\
\text { Horial }\end{array}$ & $\mathrm{R}$ & FC \\
\hline Laniidae & Lanius schach & Rufous Backed Shrike & $\begin{array}{l}\text { Badami-pith } \\
\text { Latora/Koshai }\end{array}$ & $\mathrm{R}$ & $\mathrm{R}$ \\
\hline \multirow{6}{*}{ Corvidae } & $\begin{array}{l}\text { Dendrocitta vagabunda } \\
\text { (Latham 1790) }\end{array}$ & Indian tree pie & $\begin{array}{l}\text { Horichacha, Kutum- } \\
\text { Pakhi }\end{array}$ & $\mathrm{R}$ & FC \\
\hline & $\begin{array}{l}\text { Corvus macrorhynchos } \\
\text { (Wagler, 1827) }\end{array}$ & Jungle crow & Dar Kak & $\mathrm{R}$ & FC \\
\hline & $\begin{array}{l}\text { Corvus splendens } \\
\text { (Vieillot, 1817) }\end{array}$ & House crow & Pati Kak & $\mathrm{R}$ & $\mathrm{VC}$ \\
\hline & $\begin{array}{l}\text { Oriolus xanthornus } \\
\text { (Linnaeus, 1758) }\end{array}$ & Black hooded oriole & Haldey Pakhi & $\mathrm{R}$ & FC \\
\hline & $\begin{array}{l}\text { Rhipidura albicollis } \\
\text { (Vieillot, 1818) }\end{array}$ & $\begin{array}{l}\text { White throated Fantail } \\
\text { Flycatcher }\end{array}$ & Lej Nachani & $\mathrm{R}$ & $\mathrm{R}$ \\
\hline & $\begin{array}{l}\text { Aegithina tiphia } \\
\text { (Linnaeus.1858) }\end{array}$ & Common iora & Fotikjal & $\mathrm{R}$ & FC \\
\hline $\begin{array}{c}\text { Terpsiphone } \\
\text { Paradisil } \\
\text { (Linnaeus, 1758) }\end{array}$ & Paradise Flycatcher & Shah Bulbul & $\mathrm{R}$ & $\mathrm{R}$ & \\
\hline $\begin{array}{c}\text { Dicrurus } \\
\text { macrocercus } \\
\text { (Vieillot, 1817) }\end{array}$ & $\begin{array}{l}\text { Block Drongo, } \\
\text { Kingcrow }\end{array}$ & Kalo Fingey & $\mathrm{R}$ & $\mathrm{C}$ & $\mathrm{VC}$ \\
\hline \multirow{4}{*}{$\begin{array}{c}\text { Dicrurus } \\
\text { aeneus } \\
\text { (Vieillot, 1817) } \\
\text { Dicrurus } \\
\text { hottentottus } \\
\text { (Linnaeus } \\
1766 \text { ) }\end{array}$} & Bronzed Drongo & Choto Fingey & $\mathrm{R}$ & $\mathrm{R}$ & $\mathrm{C}$ \\
\hline & Spangled Drongo & Keshraj & $\mathrm{R}$ & $\mathrm{R}$ & $\mathrm{R}$ \\
\hline & $\begin{array}{l}\text { Acridotheres fuscus } \\
\text { (Wagler, 1827) }\end{array}$ & Jungle Myna & Jhunti Shalik & $\mathrm{R}$ & $\mathrm{C}$ \\
\hline & $\begin{array}{l}\text { Acridotheres tristis } \\
\text { (Linnaeus, 1766) }\end{array}$ & Common Myna & Bhat Shalik & $\mathrm{R}$ & VC \\
\hline Hirundinidae & $\begin{array}{l}\text { Hirundo rustica } \\
\text { (Linnacus 1758) }\end{array}$ & $\begin{array}{l}\text { Barn Swallow, Common } \\
\text { Swallow }\end{array}$ & Ababil & $\mathrm{M}$ & $\mathrm{R}$ \\
\hline \multirow[t]{2}{*}{ Pycnonotidae } & $\begin{array}{l}\text { Pycnonotus jocous } \\
\text { (Linnaeus1758) }\end{array}$ & Red-whiskered Bulbul & Shipahi Bulbul & $\mathrm{R}$ & $\mathrm{C}$ \\
\hline & $\begin{array}{l}\text { Pycnonotus cafer } \\
\text { (Linnaeus 1766) }\end{array}$ & Red- vented Bulbul & Bulbuli & $\mathrm{R}$ & $\mathrm{C}$ \\
\hline \multirow[t]{4}{*}{ Muscicapidae } & $\begin{array}{l}\text { Locustella naevia } \\
\text { (Boddaent, 1783) }\end{array}$ & Grasshopper Warbler & Foring warbler & $\mathrm{R}$ & $\mathrm{R}$ \\
\hline & $\begin{array}{l}\text { Megalurus palustris } \\
\text { (Horsefield, 1821) }\end{array}$ & $\begin{array}{l}\text { Striated Marsh Warbler, } \\
\text { Striated Grassbird }\end{array}$ & Jolar Chhatare & $\mathrm{R}$ & $\mathrm{R}$ \\
\hline & $\begin{array}{l}\text { Orthotomus sutorius } \\
\text { (Pennant, 1769) }\end{array}$ & Common Tailorbird & Tuntuni & $\mathrm{R}$ & $\mathrm{C}$ \\
\hline & $\begin{array}{l}\text { Phylloscopus affinis } \\
\text { (Tickell, 1833) }\end{array}$ & Tickell's Leaf Warbler & Tickeller Pata Futki & $\mathrm{M}$ & $\mathrm{R}$ \\
\hline \multirow{3}{*}{ Nectariniidae } & $\begin{array}{l}\text { Dicaeum cruentatum } \\
\text { (Lannaeus, 1758) }\end{array}$ & $\begin{array}{l}\text { Scarlet-backed Flower } \\
\text { Pecker) }\end{array}$ & Lal Pati Fuljhuri & $\mathrm{R}$ & $\mathrm{R}$ \\
\hline & $\begin{array}{l}\text { Nectarinia zeylonica } \\
\text { (Linnaeus, 1766) }\end{array}$ & Purple rumped Sunbird & $\begin{array}{l}\text { Beguni-komor } \\
\text { Moutusi }\end{array}$ & $\mathrm{R}$ & $\mathrm{R}$ \\
\hline & $\begin{array}{l}\text { Nectarinia asiatica } \\
\text { (Latham, 1790) }\end{array}$ & Purple Sunbird & Niltuni & $\mathrm{R}$ & $\mathrm{R}$ \\
\hline Passeridae & $\begin{array}{l}\text { Passer domesticus } \\
\text { (Linnaeus, 1758) }\end{array}$ & House sparrow & Charui & $\mathrm{R}$ & VC \\
\hline \multirow{2}{*}{ Motacillidae } & $\begin{array}{l}\text { Motacilla alba } \\
\text { (Linnaeus, 1758) }\end{array}$ & White Wagtail & Sada Khonjon & $\mathrm{M}$ & $\mathrm{R}$ \\
\hline & $\begin{array}{l}\text { Motacilla flava } \\
\text { (Linnaeus,.1758) }\end{array}$ & Yellow wagtail & Halud Khonjon & $\mathrm{M}$ & $\mathrm{R}$ \\
\hline
\end{tabular}

Note: R: Resident, M: Migratory, ${ }^{*}$ Hussain (2008), ${ }^{* *} \mathrm{VC}=$ very common, $\mathrm{C}=$ common, $\mathrm{FC}=$ fairly common, $\mathrm{R}=$ rare. 
The species diversity for different habitats of the study period is presented in Table 4. It was found that majority of the species used more than one habitat. The highest number of 52 species (91.23\%) was observed to roost on trees whereas the lowest number of 3 species $(5.26 \%)$ was found to reside around ponds, thus indicating that the passerine birds were not available in habitats like ponds, ditches and water edges.

Table 2. Status of the recorded bird groups of the study area

\begin{tabular}{|c|c|c|c|c|c|c|}
\hline \multirow{2}{*}{ Status } & \multicolumn{2}{|c|}{$\begin{array}{c}\text { Non- } \\
\text { Passerine }\end{array}$} & \multicolumn{2}{c|}{ Passerine } & \multicolumn{2}{|c|}{ Total } \\
\cline { 2 - 7 } & No. & $\%$ & No. & $\%$ & No. & $\%$ \\
\hline $\begin{array}{c}\text { Very } \\
\text { Common } \\
\text { (VC) }\end{array}$ & 5 & 18.52 & 5 & 16.66 & 10 & 17.54 \\
\hline $\begin{array}{c}\text { Common } \\
\text { (C) }\end{array}$ & 7 & 25.93 & 6 & 20.00 & 13 & 22.81 \\
\hline $\begin{array}{c}\text { Fairly } \\
\text { Common } \\
\text { (FC) }\end{array}$ & 3 & 11.11 & 5 & 16.67 & 8 & 14.04 \\
\hline Rare (R) & 12 & 44.44 & 14 & 46.67 & 26 & 45.61 \\
\hline Total & 27 & 100 & 30 & 100 & 57 & 100 \\
\hline
\end{tabular}

Table 3. Species diversity of birds at different sites of the study area.

\begin{tabular}{|c|c|c|c|c|}
\hline Sites & $\begin{array}{c}\text { Non } \\
\text { Passerine } \\
\text { species }\end{array}$ & $\begin{array}{c}\text { Passerine } \\
\text { species }\end{array}$ & $\begin{array}{c}\text { Total } \\
\text { No. of } \\
\text { species }\end{array}$ & $\begin{array}{c}\% \text { of total } \\
\text { recorded } \\
\text { species }\end{array}$ \\
\hline A & 20 & 19 & 39 & 68.42 \\
\hline B & 17 & 20 & 37 & 64.91 \\
\hline C & 10 & 17 & 27 & 47.37 \\
\hline D & 11 & 14 & 25 & 43.86 \\
\hline E & 12 & 15 & 27 & 47.37 \\
\hline
\end{tabular}

$\mathrm{A}=$ Joypurhat Sadar, $\mathrm{B}=$ Khetlal, $\mathrm{C}=$ Panchbibi, $\mathrm{D}=$ Akkelpur, $\mathrm{E}=$ Kalai

Table 4. Habitat-wise species diversity of birds of the study area

\begin{tabular}{|c|c|c|c|c|}
\hline Habitats & $\begin{array}{c}\text { Non- } \\
\text { passerines }\end{array}$ & Passerines & $\begin{array}{c}\text { Total } \\
\text { No. of } \\
\text { species }\end{array}$ & $\begin{array}{c}\% \text { of } \\
\text { total } \\
\text { recorded } \\
\text { species }\end{array}$ \\
\hline Open area & 5 & 18 & 23 & 40.35 \\
\hline $\begin{array}{c}\text { Agricultural } \\
\text { land }\end{array}$ & 6 & 10 & 16 & 28.07 \\
\hline $\begin{array}{c}\text { Human } \\
\text { habitations }\end{array}$ & 2 & 6 & 8 & 14.04 \\
\hline Bush & 13 & 18 & 31 & 54.39 \\
\hline Tree & 13 & 29 & 52 & 91.23 \\
\hline Pond & 3 & 0 & 3 & 5.26 \\
\hline Ditch & 5 & 0 & 5 & 8.77 \\
\hline Water edge & 7 & 0 & 7 & 12.28 \\
\hline
\end{tabular}

The number of bird species seems to be fewer in the study area, which may be due to the short span of the study period. There might be some species which can only be seen if daily and random observations are made. Most of the published reports on the avifauna of different regions of Bangladesh are on the Sundarbans and the islands, where the shore birds constitute the major species followed by the forest birds. Ahsan and Khanom (2005) listed 92 bird species from the Chittagong University campus, among which 49 species are in common with the avifauna of Joypurhat district. The avifauna of Joypurhat therefore is quite different from that of other areas. However, the present study corroborates to the reports of Haque (1976) and Sarker et al. (2009), who showed that the number of passerine birds mostly supersede the non-passerine ones in the country (Hussain 2008, Khan R, 2008).

\section{Conclusion}

Although the study area harbours a considerable number of bird species, some potential threats to birds were identified during the study period. The major threats were illegal cutting of trees and bamboo, conversion of natural forests to monoculture plantations and agricultural fields, and hunting and trapping of birds, especially by the ethnic tribal people. The minor threats included nestling-theft for selling as cage birds and large-scale illegal harvest of forest fruits such as 'chapalish' (Artocarpus chaplasha) and 'latkan' (Bixa sp.). Some general considerations need attention for protecting birds and their habitats from above mentioned threats. It is therefore recommended that further intensive studies need to be conducted on the protection and conservation of birds in Joypurhat District in the near future.

\section{References}

Ahsan MF and Khanom N 2005. Birds of the Chittagong University Campus, Chittagong. The Chittagong Univ. J. Sci. 29(1):77-88.

Ali S. 1996. The Book of Indian Birds. Bombay Natural History Society, Oxford Univ. Press, Bombay.

Banglapedia 2003. National Encyclopedia. Asiatic Society of Bangladesh, Dhaka. 9: 470-474.

Gotelli JN. 2002. Biodiversity in the scales. Nature 419: 575.

Grewal B, Harvey B, and Pfister O. 2002. A photographic guide to the birds of India including Nepal, Sri Lanka, The Maldives, Pakistan, Bangladesh and Bhutan. Christopher Helm, London. 512 pp.

Haque AKMF. 1976. Birds of the Rajshahi University Campus. Bangladesh J. Zool. 4(2): 125-127.

Harvey B. 1990. Birds of Bangladesh. The University Press, Dhaka, Bangladesh. 
Husain KZ. 1969. Field notes on the birds of the Chittagong Hill Tract. J. Asiat. Soc. Pakistan, 13(1): 91-101.

Husain KZ. 1975. Birds of the Pablakhali Wildlife Sanctuary. Bangladesh J. Zool. 3(2):145-155.

Hussain KZ. 2008. Birds of Bangladesh: An Annotated Comparative Checklist (For the $20^{\text {th }}$ Century). Majid Publication, Dhaka. 95 pp.

Khan MAR. 1982. On the major wildlife species of St. Martin Island. Paper presented at the 6/7th Annual Bangladesh Science Conference, February, 1982, BARI, Joydebpur, Dhaka. p. 5.

Khan R. 2008a. Bangladesher Pakhi (Birds of Bangladesh, in Bangla), Bangla Academy, Dhaka. 384 pp.

Khan MMH. 2008b. Protected Areas of Bangladesh. A Guide to Wildlife Nishorgo Program, Bangladesh Forest Department, Dhaka.

Odum EP. 1971. Fundamentals of Ecology. W. B. Saunders Co., Philladelphia. 574 pp.

Sarkar, N. J., Sultana, D., M. Firoj Jaman and M. Khalilur Rahman 2009. Diversity and population of avifauna of two urban sites in Dhaka, Bangladesh. Ecoprint 16: 1-7.
Sarker SU and Sarker NJ 1986. Status and distribution of birds of the Sundarbans. J. Noami, 3(1): 19-37.

Sarker SU, Jaman MF, Hossain ML and Sarker NJ. 2001. Wildlife diversity of Moheskhali Island: Their ecology and conservation issues. J. Noami 18(1): 17-31.

Sarker SU, Jaman MF, Sarker NJ and Hossain ML. 2000a. Status of wildlife of St. Martin Island, Bangladesh. Dhaka Univ. J. Biol. Sci. 9(1): 75-85.

Sarker SU, Jaman MF, Sarker NJ and Rahman MK. 2000b. Diversity of avifauna in Bagkhali range, Cox's Bazar forest division. Bangladesh Environ. pp. 230-238.

Siddiqui NA, Shahidullah M, Shahjalal MAH. 2008. An overview of diversity, trends and threats of birds. Indian J. Forestry 14(2): 17-45.

Southwick CH. 1976. Ecology and the Quality of our Environment. D. Van Nostrand Co., New York. 426 pp.

Manuscript received on 9 November 2011 and revised on 7

December 2011 